\title{
Induction of Chromosomal Aberrations at Fluences of Less Than One HZE Particle per Cell Nucleus
}

\author{
Megumi Hada ${ }^{\mathrm{a}}$, Lori J. Chappell ${ }^{\mathrm{a}}$, Minli Wang ${ }^{\mathrm{a}}$, Kerry A. George ${ }^{\mathrm{b}}$ \\ and Francis A. Cucinotta ${ }^{\mathrm{c}}$ \\ ${ }^{a}$ Universities Space Research Association, Houston, TX, 77058 \\ ${ }^{b}$ Wyle Integrated Science and Engineering Group, Houston, TX, 77058 \\ ${ }^{c}$ University of Nevada, Las Vegas, Las Vegas, NV, 89154
}

\begin{abstract}
The assumption of a linear dose response used to describe the biological effects of high LET radiation is fundamental in radiation protection methodologies. We investigated the dose response for chromosomal aberrations for exposures corresponding to less than one particle traversal per cell nucleus by high energy and charge (HZE) nuclei. Human fibroblast and lymphocyte cells where irradiated with several low doses of $<0.1 \mathrm{~Gy}$, and several higher doses of up to $1 \mathrm{~Gy}$ with $\mathrm{O}(77 \mathrm{keV} / / \mathrm{m}), \mathrm{Si}(99 \mathrm{keV} / / \mathrm{m})$, Fe $(175 \mathrm{keV} /(\mathrm{m})$, Fe $(195 \mathrm{keV} / / \mathrm{m})$ or Fe $(240 \mathrm{keV} / / \mathrm{m})$ particles. Chromosomal aberrations at first mitosis were scored using fluorescence in situ hybridization (FISH) with chromosome specific paints for chromosomes 1, 2 and 4 and DAPI staining of background chromosomes. Non-linear regression models were used to evaluate possible linear and non-linear dose response models based on these data. Dose responses for simple exchanges for human fibroblast irradiated under confluent culture conditions were best fit by non-linear models motivated by a non-targeted effect (NTE). Best fits for the dose response data for human lymphocytes irradiated in blood tubes were a NTE model for $\mathrm{O}$ and a linear response model fit best for $\mathrm{Si}$ and Fe particles. Additional evidence for NTE were found in low dose experiments measuring $\gamma \mathrm{H} 2 \mathrm{AX}$ foci, a marker of double strand breaks (DSB), and split-dose experiments with human fibroblasts. Our results suggest that simple exchanges in normal human fibroblasts have an important NTE contribution at low particle fluence. The current and prior experimental studies provide important evidence against the linear dose response assumption used in radiation protection for HZE particles and other high LET radiation at the relevant range of low doses.
\end{abstract}

